unknown. We hypothesised that information of ischemia and atherosclerosis which can be achieved simultaneously by ultrasound (SE and carotid ultrasound) can provide incremental prognostic information in these patients.

Methods Consecutive patients with no previous history of CAD investigated with SE for suspected angina underwent a simultaneous carotid ultrasound. Carotid plaque burden was assessed. Patients were followed up for combined major adverse cardiac events (MACE) of all-cause mortality, non-fatal myocardial infarction and unplanned coronary revascularization.

Results Of the 591 patients, (269 male (46\%), mean age 59 \pm 11 years), 67 (11\%) demonstrated myocardial ischemia by SE. Prevalence of carotid plaque disease was higher (59\%) but similar in normal and abnormal SE patients $(58 \%$ vs. $66 \%$, p $=0.22$ ). At a mean of 37 months, 580 (98\%) could be followed up during which 40 MACE occurred. In the multivariable model pre-test probability of CAD $(\mathrm{p}=0.001)$, abnormal SE $(\mathrm{p}<0.0001)$ and plaque burden $(\mathrm{p}<0.0001)$ predicted MACE after adjusting for age, gender, cardiac risk factors and baseline drug therapy. MACE rate/year increased from $0.9 \%$ vs. $1.95 \%$ vs. $4.23 \%$ vs. $9.58 \%$ ( $p<0.0001$ ) in patients with no plaque and normal SE vs. presence of plaque and normal SE vs. no plaque and abnormal SE vs. plaque and abnormal SE, respectively. Figure demonstrates the prognostic value of simultaneous carotid plaque assessment and SE. When prognosis was assessed in a hierarchical manner as done clinically (pre-test probability of CAD followed by SE and carotid ultrasound), it showed significant increment in global chi square from 22.1 to 48.9 to 78.5 ( $p<0.0001$ ).

Conclusion In patients with suspected stable angina but without known CAD simultaneous SE (for ischemia) and carotid ultrasound (for atherosclerosis) provided synergistic prognostic value. This study supports simultaneous carotid ultrasound in such patients investigated by SE and has implications in primary prevention treatment.

\section{DIAGNOSTIC ACCURACY OF STRESS ECHOCARDIOGRAPHY COMPARED WITH INVASIVE CORONARY ANGIOGRAPHY WITH FRACTIONAL FLOW RESERVE FOR THE DIAGNOSIS OF HAEMODYNAMICALLY SIGNIFICANT CAD IN PATIENTS WITH KNOWN OR SUSPECTED CAD}

${ }^{1}$ Sothinathan Gurunathan* ${ }^{1}$ Grace Young, ${ }^{1}$ Guy Parsons, ${ }^{1}$ Nikos Karogiannis, ${ }^{1}$ Anastasia Vamvakidou, ${ }^{1}$ Ahmed Elghamaz, ${ }^{1,2}$ Roxy Senior. ${ }^{1}$ Department of Cardiology, Northwick Park Hospital, Harrow, UK; ${ }^{2}$ Department of Cardiology, Royal Brompton Hospital, National Heart and Lung Institute, Imperial College, London, UK; *Presenting Author

\subsection{6/heartjnl-2016-309890.132}

Introduction Haemodynamically significant coronary artery disease $(C A D)$ is an important indication for revascularisation. Wall motion analysis during stress echocardiography (SE) is a noninvasive alternative to invasive fractional flow reserve (FFR) for evaluating hemodynamically significant CAD. We sought to determine the diagnostic accuracy of SE compared with invasive coronary angiography with FFR for the diagnosis of hemodynamically significant CAD.

Methods and Results Between January 2008 and April 2015, all patients that underwent clinically indicated FFR measurements during invasive angiography and SE in close succession were analysed. Patients were excluded if tests were not done within 6 months of each other, or an intervening percutaneous coronary procedure or acute coronary syndrome occurred. 184 patients (mean age 66.5yrs, 59 (32\%) female) were identified. The majority of patients underwent coronary angiography following SE. The prevalence of known CAD, diabetes

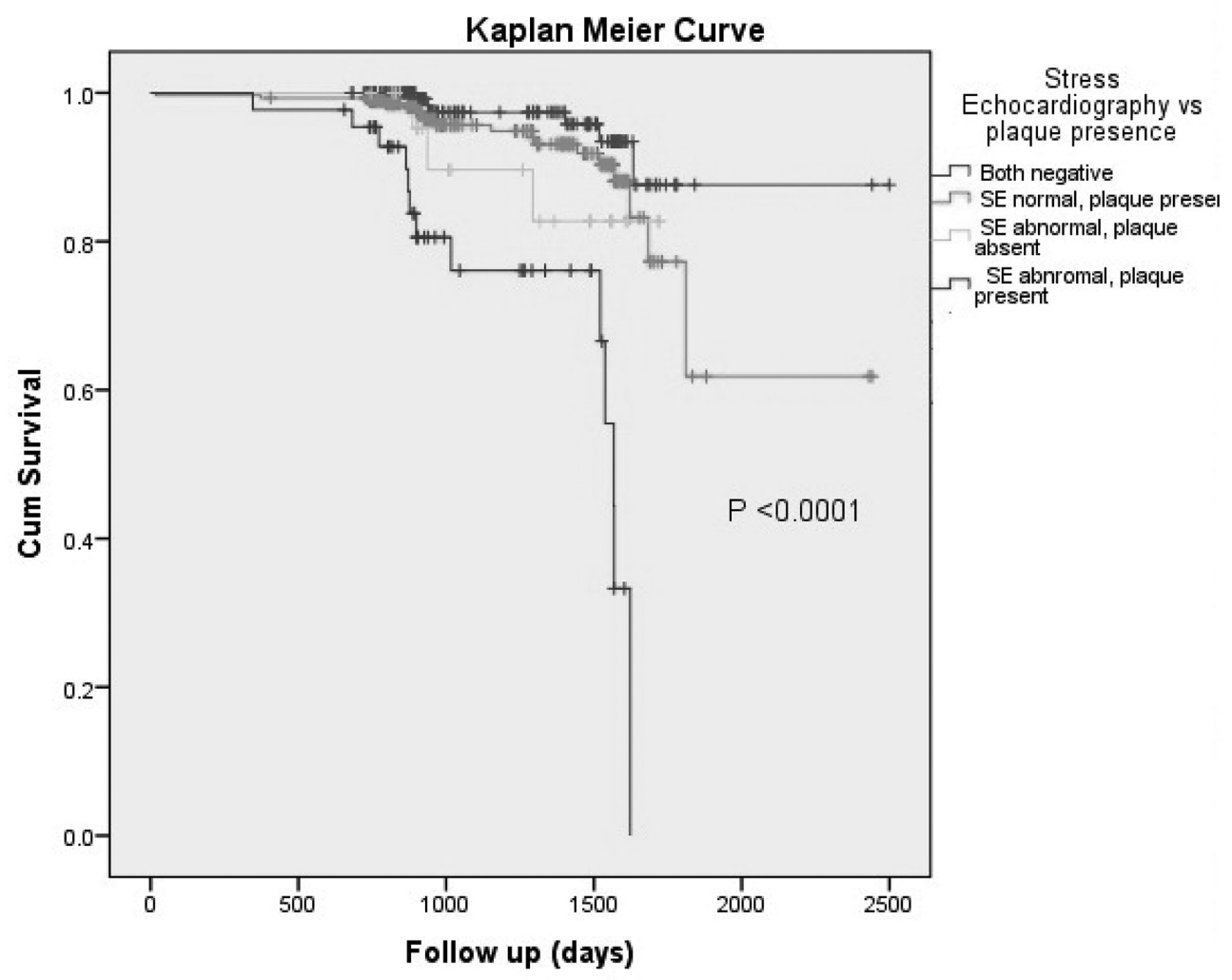

Abstract 131 Figure 1 
and chronic kidney disease were $46 \%, 43 \%$ and $13 \%$ respectively, and $14(8 \%)$ patients had previous coronary artery bypass surgery. Exercise SE was performed in 84 (46\%) patients and Dobutamine SE in 100 (54\%) patients. Contrast was used in 158 patients (86\%). In 108 patients (59\%), the SE was positive for inducible ischaemia. From 217 vessels analysed, the Left Anterior Descending Artery, Right Coronary Artery, Left Circumflex Artery and Left Main Coronary artery were involved in 120 (55\%), 47 (22\%), 30 (14\%), 18 (8\%) respectively, with 2 vessels being grafts. 46 FFR measurements were positive (21\%) and 171 were negative (79\%), using a cut off of $\leq 0.80$. At the vessel level, the sensitivity, specificity, positive predictive value (PPV) and negative predictive value (NPV) of SE for identifying significant disease as assessed by FFR was 70\%, 77\%, 45\% and 90\% respectively. In 73 patients, there was single vessel disease on angiography. At the vessel level, the sensitivity, specificity, PPV and NPV were $85 \%, 68 \%, 37 \%$ and $95 \%$.

Conclusion To date this is the largest study comparing SE and FFR for the assessment of the physiological significance of a coronary lesion, and reflects real world experience. SE demonstrates good diagnostic accuracy and excellent NPV for excluding flow-limiting disease. The low PPV is likely to represent the commencement of medical therapy following a positive $\mathrm{SE}$, as well as referral bias (since only patients with positive SE underwent angiography) as well as the low prevalence of positive FFR measurements in this population. The presence of a haemodynamically significant stenosis can be accurately ruled out with SE.

\section{ALTHOUGH CT CORONARY ANGIOGRAPHY IN THE WEST OF SCOTLAND IS USED IN A HIGHER RISK POPULATION THAN RECOMMENDED BY NICE, THE RATE OF SUBSEQUENT INVASIVE CORONARY ANGIOGRAPHY IS LOWER THAN IN THE PROMISE AND SCOT-HEART STUDIES} ${ }^{1}$ Alice M Jackson*, ${ }^{2}$ Manwella Ftouni, ${ }^{1}$ Elizabeth Philip, ${ }^{1} J a c q u e l i n e ~ A d a m s$,
${ }^{1}$ Keith Oldroyd, ${ }^{1}$ Richard Good. Byrne,
*Presenting Author

\subsection{6/heartjnl-2016-309890.133}

Background NICE guidelines recommend CT coronary angiography (CTCA) as a first line investigation for patients with chest pain and an estimated likelihood of coronary artery disease (CAD) of 10-29\%. These guidelines do not recommend exercise testing in this patient group. The recently published PROMISE and SCOT-HEART studies extended the use of CTCA to moderate and high risk patients. Neither study has shown clear clinical benefit for patient randomised to CTCA and there remains a divergence of opinion regarding the appropriateness of CTCA in these patient groups. In particular, there is a concern that CTCA may increase the number of future invasive coronary angiograms. We studied patients referred to the CTCA service in our centre and compared their predicted risk, prior stress testing and subsequent investigations to the NICE guidance and with data from the PROMISE and SCOT-Heart studies.

Methods Data was collected prospectively for consecutive patients undergoing CTCA over a 3 month period. CTCA reports were retrospectively reviewed with details recorded of the scan protocol, scan quality and severity of coronary disease. Our cardiac catheterisation database was retrospectively interrogated 6 months after the final patient underwent CTCA to document any subsequent invasive coronary angiography, PCI or CABG.

Results A total of 226 patients underwent CTCA during the 3 month study period (Table 1). The distribution of likelihood of CAD according the NICE guideline tables is shown in Figure 1 . Only $34 / 226(15 \%)$ had a pre-test probability of having significant CAD within the $10-29 \%$ category. Prior to CTCA, 153 patients (68\%) had exercise ECG testing with 12/226 (5\%) undergoing stress perfusion imaging. Overall, 164/226 (73\%) patients had normal arteries or minimal CAD with 52 (23\%) patients reported to have moderate/severe disease (Figure 2). 21/226 (9.3\%) patients subsequently underwent invasive coronary angiography. 5 of these patients had pressure wire assessment (PWS) of coronary lesions with one of these patients subsequently undergoing revascularisation by CABG. An additional 4 patients underwent percutaneous revascularisation without PWS.

Conclusion Only a minority of patients referred for CTCA to our centre were within the pre-test probability category recommended by NICE for this test. The majority of patients had undergone exercise testing prior to CTCA again suggesting limited adherence to the NICE guidelines. Despite the majority of patients having a pre-test probability $>30 \%$, only 9.3\% of patients underwent subsequent invasive angiography, less than in the patient group randomised to CTCA in both the PROMISE and SCOT-HEART studies (12\%). Further studies are needed to better define the population that would most benefit from CTCA.

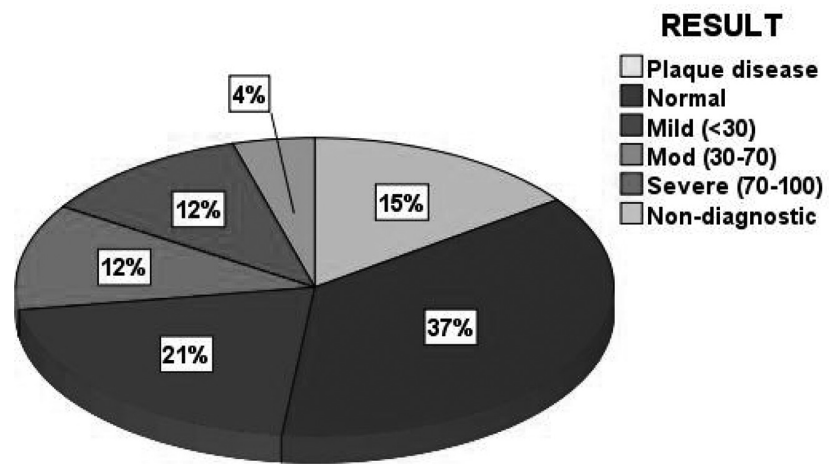

Abstract 133 Figure 2 Degree of coronary artery disease identified by CTCA

Abstract 133 Table 1 Baseline characteristics

\begin{tabular}{ll}
\hline Patient characteristic & $\mathrm{n}==226$ \\
\hline Age (mean \pm SD) & $56 \pm 11$ \\
Male (\%) & $86(38)$ \\
Dyslipidaemia (\%) & $118(52)$ \\
Diabetes (\%) & $20(9)$ \\
Smoking History (\%) & $106(47)$ \\
Chest pain assessment & \\
Non-anginal & $41(18)$ \\
Atypical & $134(59)$ \\
Typical & $51(23)$ \\
\hline
\end{tabular}

